\title{
Prevalence and correlates of neuropsychological deficits in amyotrophic lateral sclerosis
}

\author{
Paul J Massman, Jamie Sims, Norma Cooke, Lanny J Haverkamp, Vicki Appel, \\ Stanley H Appel
}

\begin{abstract}
Objective-To determine the prevalence and correlates of neuropsychological impairment in a large cohort $(n=146)$ of patients with typical, sporadic (nonfamilial) amyotrophic lateral sclerosis. Methods-A battery of neuropsychological tests was administered to patients with amyotrophic lateral sclerosis who were attending a monthly outpatient clinic or who were in hospital undergoing diagnostic tests.

Results-Comparing individual patient's scores with relevant normative data, $35.6 \%$ of the patients displayed evidence of clinically significant impairment, performing at or below the 5 th percentile on at least two of the eight neuropsychological measures. Deficits were most common in the areas of problem solving, attention/mental control, continuous visual recognition memory, word generation, and verbal free recall. Impairment was most prevalent in patients with dysarthria $(48 \cdot 5 \%)$, but $27 \cdot 4 \%$ of nondysarthric patients were also impaired. Impaired patients had more severe or widespread symptoms of amyotrophic lateral sclerosis than non-impaired patients, and had fewer years of education.

Conclusion-Neither the conventional wisdom that cognition is intact in nearly all patients with amyotrophic lateral sclerosis, nor more recent suggestions that cognition is often at least mildly impaired seems to be correct. A minority of patients with amyotrophic lateral sclerosis displayed evidence of significant impairment. Dysarthria, low education, and greater severity of motor symptoms were risk factors for impairment.
\end{abstract}

(尹 Neurol Neurosurg Psychiatry 1996;61:450-455)

Keywords: amyotrophic lateral sclerosis; neuropsychology

For many years, the conventional wisdom was that dementia occurred rarely in patients with sporadic, typical amyotrophic lateral sclerosis, and that the great majority of patients displayed little or no cognitive impairment. ${ }^{1}$ However, in several neuropathological studies, it was noted that abnormalities extended beyond cortical motor areas in some nondemented patients. ${ }^{23}$

Neuroimaging ${ }^{413}$ and neuropsychologi- $\mathrm{cal}^{14-22}$ studies have also suggested that nonmotor system cerebral dysfunction and cognitive impairment may be more prevalent in amyotrophic lateral sclerosis than previously thought. Neuropsychological studies have shown that patients with amyotrophic lateral sclerosis perform less well as a group than normal controls on tests thought to tap frontal system functioning, such as word generation, non-verbal fluency, free recall, the Wisconsin card sorting test, and the Stroop test. By contrast, patients showed normal performances on tests of naming and memory retention. Findings concerning visuoperceptual and visuospatial functioning have been inconsistent. ${ }^{1014-172021}$ Overall, these findings are most compatible with mild frontal system dysfunction rather than with an "Alzheimer's pattern" of medial temporal lobe and temporal-parietal cortical involvement. A small percentage of patients do display a more global cognitive decline, and these patients may also show more widespread cortical hypometabolism, involving the temporal and parietal lobes. ${ }^{13}$

Previous neuropsychological studies have all had sample sizes of 35 or fewer patients with amyotrophic lateral sclerosis, and only examined whether group differences between patients and controls were statistically significant. In the present study, we assessed neuropsychological functioning in a large cohort $(n=146)$ of patients whose disease progress was well characterised and examined the performances of individual patients to obtain a better estimate of the true prevalence and pattern of clinically significant impairment in these patients. We also investigated possible correlates of impairment, including age, sex, educational level, duration of symptoms, bulbar versus non-bulbar onset, dysarthria, respiratory function, overall motor impairment, and depressive symptoms.

\section{Methods}

PATIENTS

Patients were recruited from Baylor College of Medicine Methodist Hospital and from the monthly MDA-ALS outpatient clinic run by the department of neurology at Baylor College of Medicine (for a detailed description of the total Baylor amyotrophic lateral sclerosis population, see Haverkamp et $a l^{23}$ ). Laboratory tests, EMG, and muscle biopsies were performed on all patients. Histories were gathered through intensive interviews of the patients and their families. Only patients fulfilling criteria for typical, sporadic (non-familial) amy- 
otrophic lateral sclerosis were selected. ${ }^{23}$ Consistent with the El Escorial diagnostic criteria, ${ }^{24}$ patients with only upper or lower motor neuron involvement were excluded, as were patients with possible postpoliomyelitis syndrome.

Of the 158 patients who were administered the neuropsychological test battery, 146 completed testing in either all eight cognitive domains $(n=118)$ or seven of them ( $n=$ 28). The remaining 12 patients, who had missing data for two or more domains of the battery, were excluded.

\section{MEASURES}

We constructed a neuropsychological test battery to tap a broad range of cognitive functions, while attempting to minimise motor demands, particularly writing, drawing, and other manual manipulation, which would unduly handicap and stress many patients. We selected tests on which patients with amyotrophic lateral sclerosis have been found to be impaired (word generation, free recall), as well as tests on which they have displayed normal performance (naming, visuoperception). We did not administer the Wisconsin card sorting test, due to time constraints, but substituted another problem solving test, the short category test. The tests which were selected seemed to have adequate and comparable normative samples. It would be desirable to have normative data for all tests which were derived from a single, large, sample, but such data were not available (with the possible exception of the Halstead-Reitan battery, which is not appropriate for patients with amyotrophic lateral sclerosis). With limited resources, we could not conduct such a large scale normative study ourselves, but as noted by Lezak, ${ }^{25}$ the use of tests with different, but comparable, normative samples is not a serious hardship, for the normative data generally serve their purpose adequately.

Patients were given tests of problem solving (short category booklet test ${ }^{26}$ ); visuoperception (judgment of line orientation ${ }^{27}$ ); verbal learning and memory (California verbal learning test, CVLT, ${ }^{28}$ total recall across the five learning trials); visual recognition memory (continuous recognition memory test, CRMT ${ }^{29}$ total accuracy, taking into account hit rate and false positive rate); attention/mental control (verbal series attention test (VSAT, ${ }^{30}$ time to complete), which includes reciting the alphabet, counting backwards from 20 , reciting the days of the week and months of the year in forward and reverse order, counting backward from 100 by threes, and alternately saying numbers and letters in ascending order (1-A-2-B, etc); confrontation naming (Boston naming test ${ }^{31}$ ); word generation (FAS test, ${ }^{32}$ saying as many words as possible beginning with the letters $F$, $A$, and $S$ in one minute periods); psychomotor speed (symbol digit modalities test, SDMT; written and oral versions, ${ }^{33}$ number of correct responses in 90 seconds); reading of irregularly spelled words (American version of the national adult reading test, ANART $^{34}$ ), and a 118 item self report questionnaire of depres- sive symptomatology (multiscore depression inventory, MDI ${ }^{35}$ ). Thus the VSAT, FAS, and SDMT tests had a time (speed) component, and the remaining tests did not. Raw scores were converted to standard scores or percentile scores using relevant normative data, correcting for age group on all measures except the ANART; for educational level on the VSAT, FAS, and SDMT measures; and for sex on the JLO, CVLT, SDMT, and MDI measures. Normative data from the test manuals were used for all measures except the FAS test, for which an auxiliary source was used. ${ }^{36}$ A dysarthria rating (absent, mild, moderate, or severe) was given to 139 patients at the time of neuropsychological testing. Finally, 78 patients were scored with our amyotrophic lateral sclerosis rating scale ${ }^{2337}$ assessing bulbar function, respiratory function, muscle strength, and upper and lower limb function. This rating scale has been shown to reliably reflect deterioration of motor systems as amyotrophic lateral sclerosis progresses, and the rate of decrement of patients' scores is an effective predictor of survival time. ${ }^{23}$ The rating and the neuropsychological evaluation were sometimes not done close together in time, so we only included ratings of patients $(n=78)$ that were made within 90 days of the neuropsychological evaluation; the mean time interval was 23.6 days and the median time interval was 10.5 days. The patients who had ratings were significantly younger, had shorter durations of illness, and were less likely to be dysarthric than were patients without ratings. This reflects the fact that patients whose motor systems were less affected were more likely to be involved in (unsuccessful) treatment trials involving monthly clinic visits, at which times they could be given both neuropsychological testing and the amyotrophic lateral sclerosis rating. Interestingly, the groups did not differ significantly on any neuropsychological measure, and did not differ in the total number of scores in the impaired range.

\section{Results}

PATIENT CHARACTERISTICS

Table 1 shows the demographic and disease related characteristics of the study patients. On average, the patients had a relatively high level of premorbid functioning, as indicated by their mean of 13.6 years of education and above average mean ANART score (which is highly correlated with premorbid verbal intellectual ability). The men to women ratio of 1.7 is similar to previous reports. Nearly half of the patients were rated as exhibiting some dysarthria, but only $20 \cdot 1 \%$ had moderate or severe dysarthria. The mean amyotrophic lateral sclerosis motor score of 60 is indicative of mild to moderate dysfunction. ${ }^{37}$ About a quarter of patients who received an amyotrophic lateral sclerosis rating scale score showed mild respiratory dysfunction. As noted in table 1, the patients' mean T score on the MDI questionnaire was $45 \cdot 2$, which indicates that on average, patients did not report experiencing a 
Table 1 Demographic and disease related characteristics

\begin{tabular}{lcc}
\hline & No & Mean (SD) \\
\hline Age & 146 & $59 \cdot 1(12 \cdot 9)$ \\
Education (y)) & 146 & $13 \cdot 6(3 \cdot 1$ \\
ANART score & 141 & $109 \cdot 4(9 \cdot 5)$ \\
\% Men & 146 & $63 \cdot 7$ \\
Symptom duration (months) & 146 & $15 \cdot 7(14 \cdot 8)$ \\
\% Bulbar onset & 146 & $19 \cdot 2$ \\
Dysarthria & 139 & $52 \cdot 5$ \\
\% Absent & & $27 \cdot 3$ \\
\% Mild & & $15 \cdot 1$ \\
\% Moderate & $5 \cdot 0$ \\
\% Severe & 78 & $60 \cdot 1(17 \cdot 6)$ \\
\% R Rating scale score & 78 & $26 \cdot 0$ \\
MDI T score & 138 & $45 \cdot 2(7 \cdot 9)$ \\
\hline
\end{tabular}

significant number of depressive symptoms (a $T$ score of 50 signifies a score at the 50 th percentile in the normative group). Nine of the 138 patients $(6.5 \%)$ obtained a $T$ score between 61 and 83, which indicates depression of "moderate" severity" and no patient scored above 83 , indicative of "severe" depression.

\section{MEAN NEUROPSYCHOLOGICAL TEST \\ PERFORMANCES}

Table 2 shows the patients' neuropsychological test performances. Normative data for the neuropsychological measures yield percentile scores for the short category test, judgment of line orientation, and the CRMT; and $z$ scores for the remaining measures. To compare performances across measures, the $z$ scores were converted to percentile scores using the cumulative probabilities of $z$ scores in a normal distribution.

As shown in table 2, the patients, as a group, performed below expectations, obtaining mean percentile scores in the 20 s on three measures, and in the 30 s on seven measures. Considering these patients' likely above average premorbid intellectual levels (as indicated by their educational attainment and ANART scores), mean scores should have exceeded the 50th percentile, but did so on only one measure-judgment of line orientation. The patients' poorest performances occurred on word generation

Table 2 Neuropsychological test performances

\begin{tabular}{lll}
\hline & No & Mean percentile \\
\hline Line orientation & 146 & $56 \cdot 3$ \\
CVLT Recognition accuracy & 145 & $38 \cdot 6$ \\
VSAT Errors & 146 & $37 \cdot 8$ \\
Boston naming test & 146 & $37 \cdot 1$ \\
CRMT Accuracy & 143 & $35 \cdot 8$ \\
Symbol digit: oral & 143 & $34 \cdot 8$ \\
Symbol digit: written & 136 & $33 \cdot 0$ \\
Short category test & 134 & $31 \cdot 6$ \\
VSAT Time & 146 & $25 \cdot 1$ \\
CVLT Learning trials 1-5 & 145 & $22 \cdot 1$ \\
FAS Word generation & 146 & $22 \cdot 1$ \\
\hline
\end{tabular}

Table 3 Percentages of patients performing below cut off points on neuropsychological measures

\begin{tabular}{lll}
\hline & $\begin{array}{l}\text { At or below Sth } \\
\text { percentile (\% patients) }\end{array}$ & $\begin{array}{l}\text { At or below 10th } \\
\text { percentile (\% patients) }\end{array}$ \\
\hline Short category test & $26 \cdot 9$ & $35 \cdot 8$ \\
VSAT Time & $24 \cdot 0$ & $31 \cdot 5$ \\
CRMT Accuracy & $22 \cdot 4$ & $28 \cdot 1$ \\
FAS Word generation & $21 \cdot 2$ & $35 \cdot 0$ \\
CVLT Learning trials 1-5 & $20 \cdot 0$ & $29 \cdot 0$ \\
Boston naming test & $11 \cdot 7$ & $15 \cdot 8$ \\
Line orientation & $8 \cdot 2$ & $13 \cdot 0$ \\
Symbol digit & $7 \cdot 4$ & 12.5 \\
\hline
\end{tabular}

(FAS), immediate free recall performance during the five learning trials of the CVLT, and time to complete the VSAT attention/mental control tasks. By contrast with their relatively poor CVLT free recall, the group performed better on the CVLT recognition memory test, suggesting that retrieval difficulties contributed to their free recall problems. In addition, although they were slow to complete the VSAT tasks, they did not make many errors.

\section{RATES OF IMPAIRMENT ON}

NEUROPSYCHOLOGICAL MEASURES

We also tabulated the frequencies of patients who showed clinically significant deficits on eight key measures (the most important measure from each of the eight neuropsychological tests). There were five measures on which at least $20 \%$ of the patients performed at or below the 5th percentile. Using a less stringent criterion of impairment-performance at or below the 10th percentile-at least $28 \%$ of the patients scored below this cut off point on the same five measures (table 3). This set of five scores included the three measures on which the group as a whole had the lowest mean performances (word generation, CVLT immediate free recall, and VSAT time), as well as two additional measures, the short category test and CRMT accuracy. Thus on these last two measures, although the group as a whole did not perform particularly poorly, there was considerable variability, and a significant proportion of patients did show deficient performance.

As expected, few patients exhibited any notable difficulties on the tests of semantic processing (Boston naming test) and visuoperceptual processing (judgment of line orientation). Perhaps the most surprising result shown in table 3 is the low percentage of patients falling below the cut off points on the symbol digit tasks. In calculating these percentages, we labelled patients as showing impairment if they fell below the cut off point on both the written and oral versions, because we were interested in the psychomotor aspects of this task (so patients who were possibly handicapped in one response mode by their motor impairment had a chance to exceed the cut off point in the other mode). If impairment had been defined as performance at or below the 5 th percentile on the written or oral versions, the percentage of impaired patients would have risen from $7 \cdot 4$ to $19 \cdot 2$.

\section{EFFECT OF DYSARTHRIA}

We examined whether dysarthria was an important variable in mediating the likelihood of impairment on the eight key measures. Two factors could be relevant: firstly, dysarthria itself could interfere with performance on some tasks; and secondly, dysarthria could be a marker of greater cerebral involvement. We compared rates of impairment on the neuropsychological measures in the 66 patients who were rated as displaying any dysarthria (mild, moderate, or severe) with the rates in the 73 patients who were rated as displaying no dysarthria. Table 4 shows that the presence of dysarthria had a large impact on only one mea- 
Table 4 Percentages of dysarthric and non-dysarthric patients performing at or below the 5 th percentile on neuropsychological measures

\begin{tabular}{lll}
\hline & $\begin{array}{l}\text { Dysarthric } \\
\text { (\% patients) }\end{array}$ & $\begin{array}{l}\text { Non-dysarthric } \\
\text { (\% patients) }\end{array}$ \\
\hline Short category test & $30 \cdot 5$ & $25 \cdot 0$ \\
VSAT Time & $34 \cdot 8^{\star}$ & $16 \cdot 4$ \\
CRMT Accuracy & $24 \cdot 6$ & $21 \cdot 1$ \\
FAS Word generation & $22 \cdot 7$ & $19 \cdot 2$ \\
CVLT Learning trials $1-5$ & $23 \cdot 1$ & $19 \cdot 2$ \\
Boston naming test & $13 \cdot 6$ & $11 \cdot 0$ \\
Line orientation & $10 \cdot 6$ & $6 \cdot 8$ \\
Symbol digit & $10 \cdot 5^{\star}$ & $5 \cdot 6$ \\
\hline${ }^{\star P}<0 \cdot 05 ; \chi^{2}$ & &
\end{tabular}

sure, VSAT time. This is expected, as speed of verbal response (for example, saying the alphabet quickly) is important on this task. However, $16.4 \%$ of non-dysarthric patients were also impaired on this measure. Impairment rates differed significantly on only one other measure, the symbol digit modalities test, but the rates were quite low in both the dysarthric and non-dysarthric groups. Dysarthria was not associated with impairment on either confrontation naming or word generation. Also, impairment rates in the non-dysarthric group ranged from $16.4 \%$ to $25.0 \%$ on the five measures which were most impaired in the total sample.

\section{BREADTH OF IMPAIRMENT}

To investigate the breadth of individual patients' neuropsychological deficits, we tabulated the number of the eight key measures on which each patient performed at or below the 5 th percentile and at or below the 10th percentile. As table 5 shows, $64.4 \%$ of the patients exhibited little or no evidence of neuropsychological impairment across tests, scoring at or below the 5 th percentile on one or no measures. The remaining $35 \cdot 6 \%$ of patients did display some notable impairment, performing at or below the 5 th percentile on two or more of the eight measures. If a more stringent cut off point of breadth of impairment is adopted (performance at or below the 5th percentile on

Table 5 Number of key measures on which individual patients performed below cut off points

\begin{tabular}{lll}
\hline No of measures & $\begin{array}{l}\text { At or below 5th percentile } \\
\text { (\% patients) }\end{array}$ & $\begin{array}{l}\text { At or below 10th percentile } \\
\text { (\% patients) }\end{array}$ \\
\hline 0 & $36 \cdot 3$ & $20 \cdot 5$ \\
1 & $28 \cdot 1$ & $28 \cdot 8$ \\
2 & $18 \cdot 5$ & $18 \cdot 5$ \\
3 & $4 \cdot 8$ & $15 \cdot 1$ \\
4 & $6 \cdot 2$ & $6 \cdot 8$ \\
5 & $3 \cdot 4$ & $4 \cdot 1$ \\
6 & $2 \cdot 1$ & $4 \cdot 1$ \\
7 & $0 \cdot 7$ & $2 \cdot 1$ \\
\hline
\end{tabular}

Table 6 Characteristics of non-impaired and impaired patients

\begin{tabular}{lcc}
\hline & $\begin{array}{l}\text { Non-impaired } \\
\text { Mean }(S D)\end{array}$ & $\begin{array}{l}\text { Impaired } \\
\text { Mean }(S D)\end{array}$ \\
\hline Age & $60 \cdot 3(12 \cdot 5)$ & $56 \cdot 9(13 \cdot 6)$ \\
Education (y) & $14 \cdot 0^{\star}(3 \cdot 1)$ & $12 \cdot 7(2 \cdot 7)$ \\
ANART & $112 \cdot 3^{\star \star}(8 \cdot 3)$ & $103 \cdot 7(9 \cdot 1)$ \\
\% Men & $63 \cdot 2$ & $64 \cdot 7$ \\
Symptom duration (months) & $16 \cdot 1(16 \cdot 0)$ & $15 \cdot 0(12 \cdot 4)$ \\
\% Bulbar onset & $16 \cdot 8$ & $23 \cdot 5$ \\
$\%$ Dysarthric & $39 \cdot 8^{\star}$ & $60 \cdot 8$ \\
ALS Motor score & $57 \cdot 0^{\star}(12 \cdot 8)$ & $66 \cdot 5(23 \cdot 5)$ \\
\% Respiratory dysfunction & $21 \cdot 6$ & $34 \cdot 6$ \\
MDI T score & $45 \cdot 2(7 \cdot 9)$ & $47 \cdot 7(8 \cdot 9)$ \\
\hline
\end{tabular}

${ }^{\star} \mathrm{P}<0.05$, two tailed $t$ test or $\chi^{2} ; \quad{ }^{\star \star} \mathrm{P}<0.01$, two tailed $t$ test. three or more measures), then $17 \cdot 2 \%$ of patients would be considered impaired. There seemed to be variability among the impaired patients regarding which particular tests were impaired; we are currently investigating this issue using cluster analytical techniques.

When considering a milder level of impairment (performance at or below the 10 th percentile), we increased to three the number of scores which had to fall below the cut off point when classifying patients as impaired or nonimpaired (less depth, but greater breadth of impairment). Using this cut off point, $32 \cdot 2 \%$ of the patients would be classified as impaired (table 5). Using the more stringent cut off point of four or more scores at or below the 10th percentile, $17 \cdot 1 \%$ of the patients would be classified as impaired.

\section{CORRELATES OF IMPAIRMENT}

We examined patient characteristics that could be associated with neuropsychological impairment. Table 6 outlines the demographic and disease related characteristics of the 94 patients who we considered non-impaired (performance at or below the 5 th percentile on one or no measures) and the 52 patients who were considered impaired (performance at or below the 5 th percentile on two or more measures).

The groups did not differ significantly in age, sex distribution, estimated duration of motor symptoms, or bulbar versus non-bulbar onset. Despite their similar duration of symptoms, the impaired group had higher (more impaired) amyotrophic lateral sclerosis rating scale scores than the non-impaired group. Considering only the sample of 78 patients who received physical examinations and were assigned amyotrophic lateral sclerosis motor scores near the time of neuropsychological testing, mean duration was 10.4 months in the non-impaired group and 11.1 months in the impaired group, a non- significant difference $(P>0.60)$. This pattern of findings suggests that the impaired patients may have had a more rapidly progressive disease.

Table 6 shows that the non-impaired group had significantly higher educational levels and higher ANART scores, suggesting that they had somewhat higher premorbid abilities. Also, dysarthria was more prevalent in the impaired group than in the non-impaired group. However, $51.5 \%$ of dysarthric patients were not impaired, and $27 \cdot 4 \%$ of non-dysarthric patients were impaired. Although respiratory dysfunction tended to be more prevalent in the impaired than in non-impaired patients, the difference in relative frequencies was not significant $(P>0 \cdot 20)$.

Regarding the possible role of depression in causing or contributing to deficits in patients with amyotrophic lateral sclerosis, mean MDI scores did not differ significantly between the two groups (table 6). We also examined the percentages of patients who obtained MDI T scores indicative of at least "moderate" depression (> 60), and found that five of $48(10 \cdot 4 \%)$ impaired patients and four of $90(4.4 \%)$ nonimpaired patients scored in this range, a nonsignificant group difference (Fisher's exact test, 
one tailed, $P=0 \cdot 16)$. Finally, we conducted analyses of covariance with MDI score and education as the covariates, and found that all differences between the impaired and nonimpaired groups on the neuropsychological measures remained significant. These findings suggest that depressive symptomatology was not a key mediating variable associated with neuropsychological impairment.

\section{Discussion}

Most $(64.4 \%)$ of the 146 patients with amyotrophic lateral sclerosis did not display notable neuropsychological deficits, but a significant minority $(35.6 \%)$ did. Thus neither the conventional wisdom that cognition is intact in most patients with amyotrophic lateral sclerosis, nor the more recent suggestions that cognition is commonly impaired, seems to be correct.

Impaired patients exhibited the most difficulty on tests of problem solving, attention/ mental control, continuous visual recognition memory, word generation, and verbal free recall; but showed normal or near normal confrontation naming, visual perception, and delayed verbal recognition memory. This pattern of performance is most compatible with frontal system dysfunction, as indicated by recent neuroimaging studies. ${ }^{10122122}$

Patients' impairment on the continuous visual recognition memory test might suggest a memory disorder and medial temporal lobe dysfunction, but this is a difficult task that has considerable attentional demands, so attentional deficits may have contributed to deficient performance. Performance on the delayed verbal recognition memory test was good, even in the impaired patients, which indicates that the patients did not have a generalised amnesic disorder. Patients' difficulties on the free recall learning trials of the CVLT were likely due to retrieval problems and perhaps to inefficient use of active, effective learning strategies. This pattern of verbal learning and memory performance has been described in others with frontal system dysfunction, such as patients with Huntington's disease. ${ }^{38}$

The one finding which was inconsistent with frontal or subcortical involvement was the patients' relatively good performance on the symbol digit modalities test, which requires psychomotor speed, and has been reported to be impaired in patients with subcortical dysfunction, as in multiple sclerosis. ${ }^{39}$ This task does contain considerable external structure, in that the response choices are visually present at all times to the patient, who must choose among them. Patients with involvement of the frontal system can often perform relatively well on tasks in which external cues can be used to guide the response. The impaired patients with amyotrophic lateral sclerosis did perform poorly on two other tests requiring rapid responding - the VSAT and FAS word generation test. These tasks require the patient to use internal cues ("working memory") to initiate and guide performance, and patients with damage to the frontal system often have diffi- culty generating and using such internal cues. ${ }^{40}$

An additional concern is the degree to which dysarthria contributed to impaired performance on tasks requiring a verbal response. Firstly, it should be noted that dysarthria was only mild in most of the patients in whom it was identified. Secondly, we found that dysarthria was associated with a substantially increased rate of impairment on only one test, the VSAT. Impairment rates on other tasks requiring verbal responses, even rapid ones (word generation, symbol digit test: oral version), were not greatly affected by the presence of dysarthria.

Dysarthria was associated with an increased risk of being classified as impaired on our test battery, but this could reflect the possibility that dysarthria is associated with greater cerebral involvement, rather than being responsible directly for impaired performance. Over half of the dysarthric patients were not impaired, and more than a quarter of non-dysarthric patients were impaired. Thus although the presence of dysarthria increased the risk of being classified as impaired, it was far from a sufficient or necessary condition of impairment. It should be noted, however, that we did clinical ratings of dysarthria, not psychoacoustical analyses, which might detect more subtle articulation difficulties.

Greater severity of overall motor symptoms of amyotrophic lateral sclerosis was associated with likelihood of impairment. Impaired patients had more severe motor deficits than non-impaired patients, despite having similar durations of deficits. In addition, lower educational level and ANART scores were associated with greater likelihood of impairment. It might be argued that patients with higher premorbid abilities may be more likely to seek evaluation of their initial symptoms of amyotrophic lateral sclerosis more quickly, and so may be at an earlier stage in the course of disease. We found that years of education correlated negatively with duration $(r=-0.33, \quad \mathrm{P}<0.01)$. However, duration of symptoms itself did not differ significantly in the impaired and nonimpaired groups, and duration was not correlated significantly with any neuropsychological test score ( $P>0.15$ for all correlations). Patients with lower premorbid ability levels may have less "brain reserve capacity" 41 than persons with higher levels, so when these persons develop amyotrophic lateral sclerosis, they more quickly reach some critical threshold at which cognitive deficits appear. This finding parallels the evidence reported for another neurodegenerative disorder, Alzheimer's disease-namely, that low education is a risk factor for displaying the cognitive impairments characteristic of this disease. ${ }^{42} 43$

Other possible correlates of cognitive impairment, including age, sex, duration of motor symptoms, bulbar onset, and respiratory dysfunction, were not predictive of impairment. By self report, depressive symptoms (or a paradoxical absence of depressive symptoms) were not associated with neuropsychological deficits.

The characterisation of amyotrophic lateral 
sclerosis as a disease entirely confined to motor systems has long been known as an oversimplification. Although damage to other portions of the CNS and resulting functional impairment may be minor by comparison with the relentlessly devastating effects on motor function, this "incidental" damage should not be overlooked when considering either patient care or aetiology of the disease. Although cognitive impairment is measurable in only a minority of patients, we have found that minority to be a significant one. The further demonstration of a significant relation between severity of motor impairment and cognitive decline supports the hypothesis that a single underlying process may be responsible for both. The recent finding $^{44}$ of the physiological absence of the calcium binding proteins calbindin and parvalbumin in selectively vulnerable spinal motor neurons would suggest the possible absence of these same constituents in neurons mediating the frontal lobe dysfunction presented here. Regardless of the aetiological explanation, amyotrophic lateral sclerosis is not exclusively a motor neuron disease, but a disease of the nervous system, with multiple functional effects which may be masked by, or ignored because of, the overwhelming sensitivity and destruction of the motor system.

We thank the members of the amyotrophic lateral sclerosis clinic team at Baylor College of Medicine and the Methodist Hospital for their contributions to gathering these data. We are grateful to the Muscular Dystrophy Association for its continuing support. Some of this research were presented at the annual meeting of the American Academy of Neurology, Seattle, May 1995.

1 Jokelainen M. Amyotrophic lateral sclerosis in Finland. Acta Neurol Scan 1977;56:185-204.

2 Lawyer T, Netsky MG. Amyotrophic lateral sclerosis: a clinico-anatomic study of fifty-three cases. Arch Neurol Psychiatry 1953;69:171-92.

3 Brownell B, Oppenheimer DR, Hughes JT. The central nervous system in motor neuron disease. $\mathcal{f}$ Neurol Neurosurg vous system in motor neur.
Psychiatry 1970;33:338-57.

4 Poloni M, Mascherpa C, Faggi L, Rognone F, Gozzoli L. Cerebral atrophy in motor neuron disease evaluated by computed tomography. $\mathcal{f}$ Neurol Neurosurg Psychiatry 1982;45:1102-5.

5 Mitsuyama Y. Presenile dementia with motor neuron disease in Japan: clinico-pathological review of 26 cases. $\mathcal{F}$ Neurol Neurosurg Psychiatry 1984;47:953-9.

6 Dalakas MC, Hatazawa J, Brooks RA, Di Chiro G. Lowered cerebral glucose utilization in amyotrophic lateral sclerosis. Ann Neurol 1987;22:580-6.

7 Hatazawa J, Brooks MC, Dalakas MC, Mansi L, Di Chiro G. Cortical motor-sensory hypometabolism in amyotrophic lateral sclerosis: a PET study. F Comput Assist Tomogr 1988;12:630-6.

8 Ohnishi T, Hoshi H, Nagamachi S, et al. Regional cerebral blood flow study with ${ }^{12}$ I-IMP in patients with degenerative dementia. AfNR Am f Neuroradiol 1991;12:5113-20.

9 Waldemar G, Vorstrup S, Jensen TS, Johnsen A, Boysen G. Focal reductions of cerebral blood flow in amyotrophic lateral sclerosis: a [99mTc]-D,L-HMPAO SPECT study. $f$ Neurol Sci 1992;107:19-28.

10 Ludolph AC, Langen KJ, Regard M, et al. Frontal lobe function in amyotrophic lateral sclerosis: a neuropsychologic and positron emission tomography study. Acta Neurol Scand 1992;85:81-9.

11 Kew JJM, Leigh PN, Playford ED, et al. Cortical function in amyotrophic lateral sclerosis: a positron emission tomography study. Brain 1993;116:655-80.

12 Kato S, Hayashi H, Yagishita A. Involvement of the frontotemporal lobe and limbic system in amyotrophic lateral sclerosis: as assessed by serial computed tomography and magnetic resonance imaging. F Neurol Sci 1993;116:52-8.

magnetic resonance imaging. F Neurol Sci 1993;116:52-8.
13 Tanaka M, Kondo S, Hirai S, et al. Cerebral blood flow and oxygen metabolism in progressive dementia associated with amyotrophic lateral sclerosis. F Neurol Sci 1993; 120:22-8.
14 Gallassi R, Montagna P, Ciardulli C, et al. Cognitive impairment in motor neuron disease. Acta Neurol Scan 1985 71:480-4.

15 David AS, Gillham RA. Neuropsychological study of motor neuron disease. Psychosomatics 1986;27:441-5.

16 Poloni M, Capitani E, Mazzini L, Ceroni M. Neuropsychological measures in amyotrophic lateral sclerosis and their relationship with CT scan-assessed cerebral atrophy. Acto Neurol Scand 1986;74:257-60.

17 Gallassi R, Montagna P, Morreale A, et al. Neuropsychological, electroencephalogram and brain computed tomography findings in motor neuron disease. Eur Neurol 1989;29:115-20.

18 Iwasaki Y, Kinoshita M, Ikeda K, Takamiya K, Shiojima T Cognitive impairment in amyotrophic lateral sclerosis and its relation to motor disabilities. Acta Neurol Scan 1990; its relation $141-3$.

19 Iwasaki Y, Kinoshita M, Ikeda K, Takamiya K, Shiojima T Neuropsychological dysfunction in amyotrophic lateral sclerosis: relation to motor disabilities. Int $\mathcal{F}$ Neurosci 1990 54:191-5.

20 Hartikainen P, Helkala E-L, Soininen H, Riekkinen P. Cognitive and memory deficits in untreated Parkinson's disease and amyotrophic lateral sclerosis patients: a comparative study. ₹ Neural Transm 1993;6:127-37.

21 Kew JJM, Goldstein LH, Leigh PN, et al. The relationship between abnormalities of cognitive function and cerebral activation in amyotrophic lateral sclerosis: a neuropsychological and positron emission tomography study. Brain logical and positron

22 Abe K, Fujimura H, Toyooka, et al. Cognitive function in motor neuron disease [abstract]. Neurology 1995;45 (supp 4):318.

23 Haverkamp LJ, Appel V, Appel SH. Natural history of ALS in a database population: validation of a scoring system and a model for survival prediction. Brain 1995;118:707-19.

24 Swash M, Leigh N. Criteria for diagnosis of familial amyotrophic lateral sclerosis. Neuromusc Dis 1992;2:7-9.

25 Lezak MD. Neuropsychological assessment. 3rd ed. New York: Oxford University Press, 1995.

26 Wetzel L, Boll TJ. Short category test, booklet format. Manual. Los Angeles: eastern Psychological Services, 1987.

27 Benton AL, Hamsher KdeS, Varney NR, Spreen $O$. Contributions to neuropsychological assessment. New York: Oxford University Press, 1983.

28 Delis DC, Kramer JH, Kaplan E, Ober BA. California verbal learning test: research edition. New York: Psychological Corporation, 1987

29 Hannay HJ, Levin HS. The continuous recognition memory test. A manual. Houston: Neuropsychological Resources, 1988.

30 Mahurin RK, Cooke N. The verbal series attention test: normal and demented older adults. Clinical Neuropsychologist 1996;10:43-53.

31 Kaplan EF, Goodglass H, Weintraub S. Boston naming test. Philadelphia: Lea and Febiger, 1983.

32 Benton $\mathrm{AL}$, Hamsher KdeS. Multilingual aphasia examination. Iowa City: University of Iowa, 1976.

33 Smith A. Symbol digit modalities test. Manual. Los Angeles: Western Psychological Services, 1973.

34 Grober E, Sliwinski $M$. Development and validation of a model for estimating premorbid verbal intelligence in the model for estimating premorbid verbal intelligen
elderly. F Clin Exp Neuropsychol 1991;13:933-49.

35 Berndt, DJ. Multiscore depression inventory. Manual. Los Angeles: Western Psychological Services, 1986.

36 Spreen O, Strauss E. A compendium of neuropsychological tests: administration, norms, and commentary. New York: Oxford University Press, 1991.

37 Appel V, Stewart SS, Smith G, Appel SH. A rating scale for amyotrophic lateral sclerosis: description and preliminary experience. Ann Neurol 1987;22:328-33.

38 Delis DC, Massman PJ, Butters N, Salmon DP, Cermak LS, Kramer JH. Profiles of demented and amnesic patients on the California verbal learning test: implications for the assessment of memory disorders. $\mathcal{f}$ Consult Clin Psychol 1991;3:19-26.

39 Rao SM. Multiple sclerosis. In: Cummings $\mathrm{IL}$ ed. Subcortical dementia. New York: Oxford University Press, 1990:164-80.

40 Goldman-Rakic PS, Friedman HR. The circuitry of working memory revealed by anatomic and metabolic imaging. In: Levin HS, Eisenberg HM, Benton AL, eds. Frontal lobe function and dysfunction. Oxford: Oxford University Press, 1991:72-91.

41 Satz P. Brain reserve capacity on symptom onset after brain injury: a formulation and review of evidence for threshold theory. Neuropsychology 1993;7:273-95.

42 Stern Y, Alexander GE, Prohovnik I, Mayeux R. Inverse relationship between education and parietotemporal perfusion deficit in Alzheimer's disease. Ann Neurol 1992; 32:371-5.

43 Katzman R. Education and the prevalence of dementia and Alzheimer's disease. Neurology 1993;43:13-20.

44 Alexianu ME, Ho B-K, Mohamed AH, La Bella V, Smith RG, Appel SH. The role of calcium-binding proteins in selective motoneuron vulnerability in amyotrophic lateral sclerosis. Ann Neurol 1994;36:846-58. 